PROCEEDINGS OF THE AMERICAN MATHEMATICAL SOCIETY

Volume 124, Number 5, May 1996

\title{
POLYNOMIALS AND LIMITED SETS
}

\author{
PABLO GALINDO \\ (Communicated by Theodore Gamelin)
}

\begin{abstract}
We prove that scalar-valued polynomials are weakly continuous on limited sets and that, as in the case of linear mappings, every $c_{0}$-valued polynomial maps limited sets into relatively compact ones. We also show that a scalar-valued polynomial whose derivative is limited is weakly sequentially continuous.
\end{abstract}

Throughout this note $E$ and $F$ will mean infinite-dimensional complex Banach spaces. Recall that $L \subset E$ is said to be limited if every $w\left(E^{\prime}, E\right)$-null sequence is uniformly convergent to 0 on $L$. Equivalently, $L \subset E$ is limited if every linear operator $T: E \rightarrow c_{0}$ maps $L$ into a relatively compact set. Attention to limited sets has focused in studying, for instance, whether they are bounding sets for holomorphic functions (see e.g. [12], [13] and [5]) or whether they are relatively compact sets (Gelfand-Phillips spaces). Every Banach space isomorphic to a subspace of $C(K), K$ a compact sequentially compact Hausdorff space, is a Gelfand-Phillips space $([5], 4.26)$. All weakly compactly generated spaces have this property as well as every weak Asplund space. Hence separable or reflexive Banach spaces are Gelfand-Phillips spaces. (See also [7] and [17].) Of course, their intrinsic properties have also been studied (see e.g. [3] and [17]). This note deals with the interplay between polynomials and limited sets and it turns out that it is quite similar to the interplay in the linear case. For instance, it is proved that every polynomial maps limited sets into limited sets. In particular, $c_{0}$-valued polynomials map limited sets into relatively compact ones. We also study those sets whose images for $c_{0^{-}}$ valued polynomials are weakly relatively compact, that is, a polynomial analogue of Grothendieck sets. For background on polynomials and holomorphic mappings we refer to [5]. All polynomials we consider are continuous for the norm topologies. $P\left({ }^{k} E ; F\right)$ denotes the space of $k$-homogeneous $F$-valued polynomials defined on $E$. In the case $F=\mathbb{C}$ we will simply omit $\mathbb{C}$. $U_{E}$ will denote the unit ball of $E$.

Lemma 1. Let $E_{1}, E_{2}, \ldots, E_{k}$ be Banach spaces.

(i) If $L_{i} \subset E_{i}, i=1, \ldots, k$, is limited, then $L_{1} \otimes \cdots \otimes L_{k}$ is limited in $E_{1} \widehat{\otimes}_{\pi} \cdots$ $\widehat{\otimes}_{\pi} E_{k}$.

(ii) If $\left\{x_{n}^{i}\right\}$ is a limited weak Cauchy sequence in $E_{i}, i=1, \ldots, k$, and at least one of them is weakly null, then $\left\{x_{n}^{1} \otimes \cdots \otimes x_{n}^{k}\right\}$ is a weakly null sequence in $E_{1} \widehat{\otimes}_{\pi} \cdots \widehat{\otimes}_{\pi} E_{k}$.

Received by the editors June 13, 1994 and, in revised form, October 19, 1994.

1991 Mathematics Subject Classification. Primary 46G20, Secondary 46B20.

Key words and phrases. Polynomial, limited set, weakly conditionally compact set.

The author was supported in part by DGICYT pr. 91-0326 and by grant 93-081.

(C)1996 American Mathematical Society 
Proof. (i) We argue inductively on $k$. For $k=1$ the result is trivial. Assume the result is true for $k-1$. Let $G=E_{1} \widehat{\otimes}_{\pi} \cdots \widehat{\otimes}_{\pi} E_{k-1}$. Let $\left\{T_{n}\right\} \subset\left(E_{1} \widehat{\otimes}_{\pi} \cdots \widehat{\otimes}_{\pi} E_{k}\right)^{\prime}=$ $\left(G \widehat{\otimes}_{\pi} E_{k}\right)^{\prime}$ be a weak* null sequence and let $\left\{x_{n}^{i}\right\} \subset L_{i}, i=1, \ldots, k$. If $x_{n}=$ $x_{n}^{1} \otimes \cdots \otimes x_{n}^{k-1}$, the sequence $\left\{x_{n}\right\}$ is limited in $G$ by the induction hypothesis. Every $T \in\left(G \widehat{\otimes}_{\pi} E_{k}\right)^{\prime}=\mathcal{L}\left(G, E_{k}^{\prime}\right)=\mathcal{L}\left(E_{k}, G^{\prime}\right)$ is defined by $T(x \otimes y)=T(x) y=T(y) x$, where $x \in G, y \in E_{k}$. For each $y \in E_{k}$, the sequence $\left\{T_{n}(y)\right\}$ is weak* null in $G^{\prime}$. Hence the limitedness of $\left\{x_{n}\right\}$ leads to $\lim _{n} T_{n}\left(x_{n}\right) y=\lim _{n} T_{n}(y) x_{n}=0$. Thus, $\left\{T_{n}\left(x_{n}\right)\right\} \subset E_{k}^{\prime}$ is a weak ${ }^{*}$ null sequence and since $\left\{x_{n}^{k}\right\}$ is limited, we conclude that

$$
\lim _{n} T_{n}\left(x_{n} \otimes x_{n}^{k}\right)=\lim _{n} T_{n}\left(x_{n}\right) x_{n}^{k}=0 .
$$

Now, it follows that $\left\{T_{n}\right\}$ converges uniformly to 0 on $L_{1} \otimes \cdots \otimes L_{k}$. Thus $L_{1} \otimes$ $\cdots \otimes L_{k}$ is limited in $E_{1} \widehat{\otimes}_{\pi} \cdots \widehat{\otimes}_{\pi} E_{k}$.

(ii) Arguing by induction again, we may assume that the sequence $\left\{x_{n}\right\}$ where $x_{n}=x_{n}^{1} \otimes \cdots \otimes x_{n}^{k-1}$ is weakly null in $G$. For $T \in\left(E_{1} \widehat{\otimes}_{\pi} \cdots \widehat{\otimes}_{\pi} E_{k}\right)^{\prime}=\mathcal{L}\left(G, E_{k}^{\prime}\right)$ as above, we obtain that $\left\{T\left(x_{n}\right)\right\}$ is weakly null in $E_{k}^{\prime}$. Since $\left\{x_{n}^{k}\right\}$ is limited, $\lim _{n} T\left(x_{n} \otimes x_{n}^{k}\right)=\lim _{n} T\left(x_{n}\right)\left(x_{n}^{k}\right)=0$, and we are done.

In the next corollary, let $\widehat{\bigotimes}_{k, s, \pi} E$ denote the symmetric $k$-fold completed projective tensor product of $E$ with itself.

Corollary 2. Let $E$ be a Banach space.

(i) If $L \subset E$ is limited, then $\widehat{\bigotimes}_{k, s, \pi} L$ is limited in $\widehat{\bigotimes}_{k, s, \pi} E$.

(ii) If $\left\{x_{n}\right\}$ is a limited sequence weakly converging to $x \in E$, then $\left\{x_{n} \otimes \cdots \otimes x_{n}\right\}$ is weakly convergent to $x \otimes \cdots \otimes x$ in $\widehat{\bigotimes}_{k, s, \pi} E$.

(iii) If $\left\{x_{n}\right\}$ is a limited weakly Cauchy sequence in $E$, then $\left\{x_{n} \otimes \cdots \otimes x_{n}\right\}$ is a weakly Cauchy sequence in $\widehat{\bigotimes}_{k, s, \pi} E$.

Proof. (i) Since $\widehat{\bigotimes}_{k, s, \pi} E$ is a complemented subspace of $\widehat{\bigotimes}_{k, \pi} E$, the statement follows from Lemma 1(i).

(ii) Let $T \in\left(\widehat{\bigotimes}_{k, s, \pi} E\right)^{\prime}$ and $y_{n}=x_{n}-x$. If $A$ is the symmetric $k$-linear form associated to $T$ we get that

$$
\begin{aligned}
T\left(x_{n} \otimes \cdots \otimes x_{n}\right) & =A\left(x+y_{n}, \ldots, x+y_{n}\right) \\
& =A(x, \ldots, x)+A\left(x, \ldots, x, y_{n}\right)+\cdots+A\left(y_{n} \ldots, y_{n}\right) .
\end{aligned}
$$

Since $\left\{y_{n}\right\}$ is weakly null, we may apply Lemma 1 (ii) with $x \in E$ fixed to show that $T\left(x_{n} \otimes \cdots \otimes x_{n}\right) \rightarrow T(x \otimes \cdots \otimes x)$ as $n \rightarrow \infty$.

(iii) Let $T \in\left(\widehat{\bigotimes}_{k, s, \pi} E\right)^{\prime}$. Assume that $\left\{T\left(x_{n} \otimes \cdots \otimes x_{n}\right)\right\}$ is not a Cauchy sequence in $\mathbb{C}$. Then there is $\varepsilon>0$ and two subsequences $\left\{x_{n_{j}}\right\}$ and $\left\{x_{m_{j}}\right\}$ such that $\left|T\left(x_{n_{j}} \otimes \cdots \otimes x_{n_{j}}\right)-T\left(x_{m_{j}} \otimes \cdots \otimes x_{m_{j}}\right)\right|>\varepsilon$ for all $j \in \mathbb{N}$. Put $b_{j}=x_{n_{j}}-x_{m_{j}}$. If $A$ is the associated symmetric $k$-linear form we get that

$$
\begin{aligned}
T\left(x_{n_{j}} \otimes \cdots \otimes x_{n_{j}}\right) & -T\left(x_{m_{j}} \otimes \cdots \otimes x_{m_{j}}\right) \\
& =A\left(x_{m_{j}}, \ldots, x_{m_{j}}, b_{j}\right)+\cdots+A\left(x_{m_{j}}, b_{j}, \ldots, b_{j}\right) .
\end{aligned}
$$

Since $\left\{x_{n}\right\}$ is weakly Cauchy, $\left\{b_{j}\right\}$ is a weakly null sequence in $E$, hence by Lemma 1(ii), $\left\{x_{m_{j}} \otimes{ }^{i} x_{m_{j}} \otimes b_{j}{ }^{k-i} \cdot \otimes b_{j}\right\}$ is weakly null in $E \widehat{\otimes}_{\pi} \cdots \widehat{\otimes}_{\pi} E$, so

$$
\lim _{j} A\left(x_{m_{j}}^{i}, b_{j}^{k-i}\right)=0 \quad \text { if } 1 \leq i<k \text {. }
$$


Thus

$$
\lim _{j} T\left(x_{n_{j}} \otimes \cdots \otimes x_{n_{j}}\right)-T\left(x_{m-j} \otimes \cdots \otimes x_{m_{j}}\right)=0 .
$$

A contradiction.

We recall that limited sets in Banach spaces are conditionally weakly compact (i.e., any sequence has a weakly Cauchy subsequence) [3]. We need the following result (see Theorem 2.6 [11]): If $B$ is a conditionally weakly compact subset of $E$, then every point in the weak closure of $B$ is the weak limit of a sequence of points in $B$.

Theorem 3. Every polynomial, $P: E \rightarrow \mathbb{C}$, is weakly continuous on limited sets.

Proof. Let $L \subset E$ be a limited set. To prove that $P: L \rightarrow \mathbb{C}$ is weakly continuous, it suffices to check that $P\left(\operatorname{cl}_{w}(B)\right) \subset \operatorname{cl}_{\mathbb{C}} P(B)$ for each $B \subset L$. Since every $a \in L$ which is in the weak closure of $B$ is the weak limit of a sequence $\left\{a_{n}\right\} \subset B$, we have by Corollary 2(ii) that $\lim _{n} P\left(a_{n}\right)=P(a)$ because there is $T \in\left(\widehat{\bigotimes}_{k, s, \pi} E\right)^{\prime}$ such that $P(x)=T(x \otimes \cdots \otimes x)$. This completes the proof.

In general the assumption on limitedness can neither be dropped nor replaced by weak compactness. (Think for instance of $E=l^{2}, P(x)=\sum x_{n}^{2}$, and the unit basis $\left\{e_{n}\right\}$ in $l^{2}$.)

Remark 4. Let $E$ be the dual of a Banach space $Y$. A subset $L \subset E$ is called $Y$ limited (see [4]) if every weakly null sequence in $Y$ is uniformly convergent to 0 on $L$. Of course, every limited set in $E$ is $Y$-limited. The ideas in the previous results allow us to show that any weak* separately continuous $k$-linear form on $E$ is weak* sequentially continuous on $Y$-limited sets. Indeed, let $B: E \times \cdots \times E \rightarrow \mathbb{C}$ be a weak $^{*}$ separately continuous $k$-linear form on $E$, and let $\left\{a_{j}^{1}\right\},\left\{a_{j}^{2}\right\}, \ldots,\left\{a_{j}^{k}\right\}$ be $Y$ limited weakly ${ }^{*}$ convergent sequences in $E$ to $a^{1}, a^{2}, \ldots, a^{k} \in E$. Put $b_{j}^{i}=a_{j}^{i}-a^{i}$; then

$$
\begin{aligned}
B\left(a_{j}^{1},\right. & \left.a_{j}^{2}, \ldots, a_{j}^{k}\right)-B\left(a^{1}, a^{2}, \ldots, a^{k}\right) \\
& =B\left(b_{j}^{1}+a^{1}, b_{j}^{2}+a^{2}, \ldots, b_{j}^{k}+a^{k}\right)-B\left(a^{1}, a^{2}, \ldots, a^{k}\right) \\
& =B\left(b_{j}^{1}, b_{j}^{2}, \ldots, b_{j}^{k}\right)+\sum B\left(b_{j}^{1}, \ldots, a^{i}, \ldots\right)
\end{aligned}
$$

where in the last sum every term has at least one of the variables equal to some $a^{i}$. If we argue by induction, each of the terms in the sum can be seen as a weak ${ }^{*}$ separately continuous $n$-linear form with $n<k$, hence $\lim _{j} B\left(b_{j}^{1}, \ldots, a^{i}, \ldots\right)=0$. For the remaining term, $B\left(b_{j}^{1}, b_{j}^{2}, \ldots, b_{j}^{k}\right)$, the mapping $x \in E \rightsquigarrow B\left(x, b_{j}^{2}, \ldots, b_{j}^{k}\right)$ is weak ${ }^{*}$ continuous, so there is $y_{j} \in Y$ such that $\left\langle y_{j}, x\right\rangle=B\left(x, b_{j}^{2}, \ldots, b_{j}^{k}\right)$ and again by the induction hypothesis, $\lim _{j}\left\langle y_{j}, x\right\rangle=\lim _{j} B\left(x, b_{j}^{2}, \ldots, b_{j}^{k}\right)=0$, because $B(x, \ldots)$ is a weak ${ }^{*}$ separately continuous $(k-1)$-linear form for each fixed $x \in E$. Therefore,

$$
\lim _{j} B\left(a_{j}^{1}, a_{j}^{2}, \ldots, a_{j}^{k}\right)=B\left(a^{1}, a^{2}, \ldots, a^{k}\right) .
$$

This remark is related to the question (settled in [1]) of finding conditions which guarantee that a weak ${ }^{*}$ separately continuous multilinear form on $E$ is weak* continuous on bounded sets. So, if $Y$ is a separable Schur space, $U_{E}$ is a $Y$-limited and weak $^{*}$ metrizable bounded set in $E$ and therefore every weak ${ }^{*}$ separately continuous multilinear form on $E$ is weak ${ }^{*}$ continuous on bounded sets. Let us also mention 
that the same type of reasoning shows that if in $\left(E, w^{*}\right)$ the bilinear separately continuous forms are continuous at 0 on bounded sets, then every separately continuous multilinear form continuous at 0 on bounded sets is continuous on bounded sets.

Theorem 5. If $L \subset E$ is a limited set and $\left\{P_{n}\right\}$ is a sequence in $P^{k}(E)$ pointwise convergent to 0 , then $\left\{P_{n}\right\}$ is uniformly convergent to 0 on $L$.

Proof. Let $T_{n} \in\left(\widehat{\bigotimes}_{k, s, \pi} E\right)^{\prime}, n \in \mathbb{N}$, be the associated linear maps to the sequence $\left\{P_{n}\right\}$. Since $T_{n} \rightarrow 0$ in $w\left(\left(\widehat{\bigotimes}_{k, s, \pi} E\right)^{\prime}, \bigotimes_{k, s, \pi} E\right)$ and the set $\left\{T_{n}\right\}$ is equicontinuous, we have that $T_{n} \rightarrow 0$ in $w\left(\left(\bigotimes_{k, s, \pi} E\right)^{\prime}, \widehat{\bigotimes}_{k, s, \pi} E\right)$. Now the result follows by using Corollary 2(i).

Corollary 6. If $L \subset E$ is limited and $P \in P\left({ }^{k} E, F\right)$, then $P(L)$ is a limited set in $F$.

Proof. Let $\left\{\phi_{n}\right\} \subset F^{\prime}$ be a $w\left(F^{\prime}, F\right)$-null sequence. Since $\left\{\phi_{n} \circ P\right\} \subset P\left({ }^{k} E\right)$ is pointwise convergent to 0 , it follows from Theorem 5 that $\left\{\phi_{n} \circ P\right\}$ converges uniformly to 0 on $L$, hence $\left\{\phi_{n}\right\}$ converges uniformly to 0 on $P(L)$. Therefore $P(L)$ is limited.

Proposition 7. If $P \in P\left({ }^{k} E, F\right)$ is weakly sequentially continuous on limited sets and $L \subset E$ is limited, then $P$ is weakly continuous in $L$ and $P(L)$ is a relatively compact set.

Proof. The continuity statement follows in the same way as Theorem 3. Let us prove that $P(L)$ is a relatively compact set. Let $A$ be the symmetric $k$-linear mapping associated to $P$. By the polarization formula and the fact that finite sums of limited sets are limited sets, we have that $A$ is weakly continuous on limited sets and furthermore we can repeat the arguments given in ([2], Lemma 2.4) to show that if $\left\{a_{j}^{1}\right\},\left\{a_{j}^{2}\right\}, \ldots,\left\{a_{j}^{k}\right\}$ are limited weakly Cauchy sequences in $E$ and at least one of them is weakly null, then the sequence $\left\{A\left(a_{j}^{1}, a_{j}^{2}, \ldots, a_{j}^{k}\right)\right\}$ converges to 0 in $F$.

Let $\left\{a_{n}\right\}$ be an arbitrary sequence in $L$. We will prove that $\left\{P\left(a_{n}\right)\right\}$ has a Cauchy subsequence in $F$. Since $L$ is weakly conditionally compact, $\left\{a_{n}\right\}$ has a weakly Cauchy subsequence $\left\{a_{n_{j}}\right\}$. The same argument as in Corollary 2(iii) shows that $\left\{P\left(a_{n_{j}}\right)\right\}$ is a Cauchy sequence in $F$.

Note that the above proposition applied to the identity map on $E$ leads to the well-known fact [8] that if every limited weakly null sequence in $E$ is norm null, then $E$ is a Gelfand-Phillips space.

Recall the following definition due to Farmer and Johnson [9]: A Banach space $E$ is said to be a $P_{N^{-}}$Schur space $(N \in \mathbb{N})$ if any sequence $\left\{a_{n}\right\} \subset E$ is a null sequence in $E$ provided that $\left\{P\left(a_{n}\right)\right\}$ converges to 0 in $\mathbb{C}$ for all polynomials $P \in P\left({ }^{N} E\right)$.

Proposition 8. Every $P_{N}$-Schur space is a Gelfand-Phillips space.

Proof. It is enough to check that every limited weakly null sequence is convergent to 0 , and this follows at once from Corollary 2(ii).

A polynomial $P \in P\left({ }^{k} E, F\right)$ is called limited (resp. weakly compact, weakly conditionally compact) if it maps the unit ball of $E$ into a limited (resp. weakly relatively compact, weakly conditionally compact) set in $F$. It is known [15] that 
for every weakly compact (resp. weakly conditionally compact) polynomial $P \in$ $P\left({ }^{k} E, F\right)$ there is a Banach space $F_{P}$ reflexive (resp. non-containing copies of $l^{1}$ ) and a polynomial $P_{1} \in P\left({ }^{k} E, F_{P}\right)$ such that $P=i \circ P_{1}$, where $i: F_{P} \rightarrow F$ is a linear operator. As a consequence of Corollary 6 and the fact that limited sets are relatively compact in reflexive Banach spaces and relatively weakly compact in spaces without copies of $l^{1}[3]$, we have the following.

Corollary 9. If $P \in P\left({ }^{k} E, F\right)$ is weakly compact (resp. weakly conditionally compact) and $L \subset E$ is limited, then $P(L)$ is a relatively compact (resp. weakly relatively compact) subset of $F$. If, moreover, $Q \in P\left({ }^{m} F, G\right)$ is limited, then $Q \circ P$ is a compact (resp. weakly compact) polynomial.

The second part of the above corollary slightly generalizes the result of Lindström stating that the composition of two limited operators is weakly compact $([16])$.

Corollary 10. If the derivative polynomial of $P: E \rightarrow \mathbb{C}, d P: E \rightarrow E^{\prime}$, is a limited polynomial, then $P$ is weakly sequentially continuous.

Proof. We may suppose $P$ is $m$-homogeneous. Recall that the derivative $d P$ is given by $d P: E \rightarrow E^{\prime}, d P(x)(u)=m A(u, x, \ldots, x), x, u \in E$, where $A$ is the symmetric $m$-linear mapping associated to $P$, and therefore, the symmetric $(m-1)$-linear mapping associated to $d P, \dot{A}$, is $\dot{A}\left(x_{1}, \ldots, x_{m-1}\right)(u)=m A\left(u, x_{1}, \ldots, x_{m-1}\right)$. We show the result by induction on the degree of $P$. It is obviously true for $m=1$. Assume it is true for all $i \in \mathbb{N}, i<m$, and let us check it for $m$. Let $\left\{a_{n}\right\} \subset E$ be a weakly convergent sequence to $a \in E$. To prove that $P(a)=\lim _{n} P\left(a_{n}\right)$, it is enough to prove that $\lim _{n} A\left(\left(a_{n}-a\right)^{i}, a^{m-i}\right)=0$ for all $i \in \mathbb{N}, i \leq m$. For $i \in \mathbb{N}, i \leq m$, consider $P_{i}: E \rightarrow \mathbb{C}$ defined by $P_{i}(x)=A\left(x^{i}, a^{m-i}\right)$, whose associated symmetric $i$-linear form is $A\left(x_{1}, \ldots, x_{i}, a^{m-i}\right)$ and whose derivative $d P_{i}$ is given by $d P_{i}(x)(u)=i A\left(u, x^{i-1}, a^{m-i}\right)$. Since $d P$ is limited, it maps any bounded set into a limited set in $E^{\prime}$ and since $\dot{A}$ shares with it this property, it follows that every $d P_{i}$ is limited. By the induction hypothesis each $P_{i}, i<m$, is weakly sequentially continuous, hence $\lim _{n} P_{i}\left(a_{n}-a\right)=\lim _{n} A\left(\left(a_{n}-a\right)^{i}, a^{m-i}\right)=0$. Moreover, $\lim _{n} P\left(a_{n}-a\right)=\lim _{n} d P\left(a_{n}-a\right)\left(a_{n}-a\right)=0$ because the set $\left\{d P\left(a_{n}-a\right): n \in\right.$ $\mathbb{N}\} \subset E^{\prime}$ is limited and the sequence $\left\{\left(a_{n}-a\right)\right\}$ is $w\left(E^{\prime \prime}, E^{\prime}\right)$-null.

Remark 11. (1) The converse to the above proposition does not hold in general. For instance, consider $E=c_{0} \times l_{1}$ and $A: E \times E \rightarrow \mathbb{C}$ defined by $A((x, \alpha),(y, \beta))=$ $\frac{\alpha(y)+\beta(x)}{2}$ where $x, y \in c_{0}$ and $\alpha, \beta \in l_{1}$. $A$ is a symmetric bilinear form whose associated polynomial, $P$, is $P((x, \alpha))=\alpha(x)$. $P$ is weakly sequentially continuous: Indeed, let $\left\{\left(x_{n}, \alpha_{n}\right)\right\}$ be a sequence in $E$ weakly convergent to $(x, \alpha)$; then $\left\{x_{n}\right\} \subset$ $c_{0}$ is weakly convergent to $x$ and $\left\{\alpha_{n}\right\} \subset l_{1}$ is weakly, hence norm, convergent to $\alpha$. Now,

$$
\begin{aligned}
& \left|P\left(\left(x_{n}, \alpha_{n}\right)\right)-P((x, \alpha))\right|=\left|\alpha_{n}\left(x_{n}\right)-\alpha(x)\right|=\left|\alpha_{n}\left(x_{n}\right)-\alpha\left(x_{n}\right)+\alpha\left(x_{n}\right)-\alpha(x)\right| \\
& \quad \leq\left|\left(\alpha_{n}-\alpha\right)\left(x_{n}\right)\right|+\left|\alpha\left(x_{n}-x\right)\right| \leq \| \alpha_{n}-\alpha|||| x_{n}||+\left|\alpha\left(x_{n}-x\right)\right|
\end{aligned}
$$

and both last sequences converge to 0 . On the other hand, $d P((x, \alpha))=(\alpha, x)$, hence $d P\left(U_{E}\right) \supset U_{l_{1}} \times U_{c_{0}}$. Consequently, $d P\left(U_{E}\right)$ cannot be limited in $E^{\prime}=l_{1} \times l_{\infty}$, because then its projection into $l_{1}$ would be a limited set containing the unit ball of $l_{1}$. 
(2) From the examples of limited nonbounding sets found by Josefson [13] and Schlumprecht [19], it follows that holomorphic mappings do not map limited sets into limited sets in general. In fact, every $f \in H(E, F)$ maps limited sets into limited sets if, and only if, every limited set in $E$ is a bounding set.

As an application of our former results, we can show the following.

Proposition 12. Let $f \in H(E) . \quad f$ is bounded on limited sets if, and only if, $f$ maps limited weakly Cauchy sequences into convergent ones. Under these conditions $f$ is weakly continuous on limited sets.

Proof. $(\Rightarrow)$ Since $f$ is bounded on limited sets, its Taylor series expansion about 0 is uniformly convergent to $f$ on limited sets as a consequence of Cauchy's inequalities. Therefore on any weakly Cauchy limited sequence, $\left\{a_{n}\right\}$, given $\varepsilon>0$, there is a polynomial, $P$, such that $\|f-P\|_{L}<\varepsilon$, where $L=\left\{a_{n} \mid n \in \mathbb{N}\right\} \subset E$. Since $\left\{a_{n}\right\}$ is weakly Cauchy, by Corollary 2(iii), there is $p \in \mathbb{N}$ such that $\left|P\left(a_{n}\right)-P\left(a_{m}\right)\right|<\varepsilon$ if $n, m>p$. Thus if $n, m>p$, then $\left|f\left(a_{n}\right)-f\left(a_{m}\right)\right|<3 \varepsilon$. Hence $\left\{f\left(a_{n}\right)\right\}$ is a Cauchy sequence. Moreover, since $f$ can be uniformly approximated by polynomials on limited sets, applying Theorem 3 , it follows that $f$ is weakly continuous on limited sets.

$(\Leftarrow)$ Just recall that limited sets are weakly conditionally compact sets.

Remark 13. (i) As already mentioned, a limited set is not necessarily a bounding set. Nevertheless, the limited sets are the (balanced) bounding sets for a smaller class of entire functions, namely, those characterized in the above proposition. Note that if we replace limited by compact, the (balanced) bounding sets for $H(E)$ do not necessarily coincide with the compact sets. Let's prove now our statement concerning limited sets: Let $B$ be a balanced bounding set in $E$ for holomorphic functions bounded on limited sets. To see that $B$ is limited, let $\left\{f_{n}\right\} \subset E^{\prime}$ be a sequence pointwise convergent to 0 . For any $\varepsilon>0$, the function $f: E \rightarrow \mathbb{C}$ defined by $f(x):=\sum_{n=0}^{\infty}\left(\frac{f_{n}}{\varepsilon}\right)^{n}(x)$ is easily seen to be bounded on limited sets, hence it is bounded on $B$. Therefore by Cauchy's inequality, $\left\|\left(\frac{f_{n}}{\varepsilon}\right)^{n}\right\|_{B} \leq\|f\|_{B}$, hence $\left\|\frac{f_{n}}{\varepsilon}\right\|_{B} \leq\|f\|_{B}^{\frac{1}{n}}$. Consequently, $\left\|f_{n}\right\|_{B} \leq 2 \varepsilon$ for sufficiently large $n$.

(ii) Whenever the limited sets are relatively weakly compact, for instance when $E$ does not contain a copy of $l_{1}$, the holomorphic functions which are bounded on limited sets are those which are weakly continuous on limited sets.

A related notion to that of limited set is the one of Grothendieck set [14]: $A \subset E$ is called a Grothendieck set if for every operator $T: E \rightarrow c_{0}, T(A)$ is a weakly relatively compact set in $c_{0}$. In view of Corollary 6 , we may ask if polynomials map Grothendieck sets into Grothendieck sets. The negative answer to this question is provided by González and Gutiérrez in [10] who show the existence of a polynomial $P: \quad l^{\infty} \rightarrow c_{0}$ which maps the unit ball of $l^{\infty}$ into a non-weakly relatively compact set. Our next proposition is a refinement of their result and its proof goes back to Proposition 4 of [3]. We will call a subset $A$ of $E$ a $P$-Grothendieck set if $P(A) \subset c_{0}$ is weakly relatively compact for every polynomial $P: E \rightarrow c_{0}$.

Proposition 14. Every P-Grothendieck set is weakly conditionally compact.

Proof. Let $A \subset E$ be a $P$-Grothendieck set and suppose it is not weakly conditionally compact. Then there is a sequence $\left\{a_{n}\right\} \subset A$ equivalent to the unit basis of $l^{1}$. As in ([6], p. 223) we can construct an operator $N: E \rightarrow L_{\infty}[0,1]$ such that $N\left(a_{n}\right)=r_{n}$ where $\left\{r_{n}\right\}$ is the sequence of Rademacher functions. Let 
$B: L_{\infty}[0,1] \times L_{\infty}[0,1] \rightarrow c_{0}$ be defined by

$$
B(f, g)=\sum_{n=1}^{\infty}\left(\int f r_{n}\right)\left(\int g r_{n}\right) u_{n}
$$

where $u_{n}=(1,1, . \stackrel{n}{.}, 1,0,0, \ldots), f, g \in L_{\infty}[0,1]$. Since $\left\{r_{n}\right\}$ is an orthonormal set, applying Bessel's inequality, we have $\left(\int h r_{n}\right)_{n} \in l^{2}$ and $\left\|\left(\int h r_{n}\right)_{n}\right\|_{2} \leq\|h\|_{2} \leq\|h\|_{\infty}$ for all $h \in L_{\infty}[0,1]$. Thus $\|B(f, g)\| \leq\|f\|_{\infty}\|g\|_{\infty}$ and $B$ is a continuous bilinear map. Therefore $Q(f)=B(f, f)$ is a 2-homogeneous polynomial and $Q \circ N$ is a 2-homogeneous polynomial on $E$. By assumption $(Q \circ N)(A)$ is a weakly relatively compact set in $c_{0}$ which contains the sequence $\left\{u_{n}\right\}$. A contradiction.

Although every weakly relatively compact set is Grothendieck, it is not necessarily $P$-Grothendieck as it follows from considering $P: l^{2} \rightarrow c_{0}$ defined by $P(x)=\sum x_{n}^{2} u_{n}$ where $x=\left(x_{n}\right) \in l^{2}, u_{n}=(1,1, . . ., 1,0,0, \ldots)$ and the unit basis $\left\{e_{n}\right\}$ in $l^{2}$, for which $\left\{P\left(e_{n}\right)\right\}$ is not a weakly relatively compact set. On the other hand, since any scalar valued polynomial on $c_{0}$ is weakly sequentially continuous, the weakly relatively compact sets in $c_{0}$ are $P$-Grothendieck. Obviously from Corollary 6 , every limited set is $P$-Grothendieck. It follows from linearization techniques (see [18]) that whenever $P\left({ }^{m} E\right)$ is reflexive, for instance if $E$ is Tsirelson's space, the unit ball is a (non-limited) $P$-Grothendieck set. We remark that any continuous polynomial maps $P$-Grothendieck sets into $P$-Grothendieck sets.

Proposition 15. Let $f: E \rightarrow F$ be a holomorphic mapping which is bounded on bounded sets in $E$. If $A \subset E$ is P-Grothendieck (resp. limited), then $f(A)$ is a $P$-Grothendieck (resp. limited) set.

Proof. We begin with the case $F=c_{0}$. Given $\varepsilon>0$ we can obtain from the Taylor series expansion of $f$ about $0 \in E$ a polynomial $P$ such that $\|f-P\|_{A}<\varepsilon$, i.e., $f(A) \subset P(A)+\varepsilon U_{c_{0}} \subset \operatorname{cl}_{w}(P(A))+\varepsilon U_{c_{0}}$, where $U_{c_{0}}$ denotes the closed unit ball of $c_{0}$. Since $A$ is $P$-Grothendieck, $P(A)$ is $P$-Grothendieck, hence weakly relatively compact. Therefore, $\operatorname{cl}_{w}(P(A))+\varepsilon U_{c_{0}}$ is a weakly closed set, so it follows that $\mathrm{cl}_{w}(f(A)) \subset \mathrm{cl}_{w}(P(A))+\varepsilon U_{c_{0}}$. Finally, we apply a lemma of Grothendieck (see [6], Lemma 2, p. 227) to prove that $\mathrm{cl}_{w}(f(A))$ is weakly compact.

For the general case, consider $P: F \rightarrow c_{0}$. By the above case, $(P \circ f)(A)$ is weakly relatively compact. Thus $f(A)$ is $P$-Grothendieck.

A similar argument holds for limited sets.

\section{ACKNOWLEDGMENT}

This paper was written during the academic year 1993-94 while I was visiting the Department of Mathematics of Kent State University. Thanks are given to the people there for their hospitality. I am especially grateful to Richard M. Aron for the helpful conversations we had during the preparation of this note. I also thank the referee for suggesting the tensor product approach.

\section{ADDED IN PROOF}

J. Gutiérrez pointed out that Theorem 3 also follows from Theorem 3.5 in his joint article with M. González, Weakly continuous mappings on Banach spaces with Dunford-Pettis property, J. Math. Anal. Appl. 173 (1993), 470-482. 


\section{REFERENCES}

1. R. Aron, B. Cole, and T. Gamelin, Weak-star continuous analytic functions, Canad. J. Math. 47 (1995), 673-683.

2. R. Aron, C. Hervés, and M. Valdivia, Weakly continuous mappings on Banach spaces, J. Funct. Anal. 52, (1983), 189-204. MR 84g:46066

3. J. Bourgain and J. Diestel, Limited operators and strict cosingularity, Math. Nachr. 11, (1984), 55-58. MR 86d:47024

4. J. Dieudonné, Sur les espaces de Köthe, J. Analyse Math. 1, (1951), 81-115. MR 12:834b

5. S. Dineen, Complex analysis in locally convex spaces, North-Holland Math. Stud., vol. 57, North-Holland, Amsterdam and New York, 1980. MR 84b:46050

6. J. Diestel, Sequences and series in Banach spaces, Graduate Texts in Math., vol. 92, SpringerVerlag, Berlin and New York, 1984. MR 85i:46020

7. L. Drewnowski, On Banach spaces with the Gelfand-Phillips property I, Math. Z. 19, (1986), 405-411. MR 88d:46024

8. L. Drenowski and G. Emmanuele, On Banach spaces with the Gelfand-Phillips property II, Rend. Cir. Mat. Palermo Ser. II 38, (1989), 377-391. MR 92a:46016

9. J. Farmer and W. Johnson, Polynomial Schur and polynomial Dunford-Pettis properties, Contemp. Math., vol. 144, Amer. Math. Soc., Providence, RI, 1993, pp. 95-105. MR 94e:46078

10. M. González and J. Gutiérrez, Unconditionally converging polynomials on Banach spaces, Math. Proc. Cambridge Philos. Soc. 117 (1995), 321-331. CMP95:05

11. The compact weak topology on a Banach space, Proc. Roy. Soc. Edinburgh Sect. A 12, (1992), 367-379. MR 93c: 46022

12. B. Josefson, Bounding sets in $l^{\infty}(A)$, J. Math. Pures Appl. 57, (1975), 397-421. MR 81a:46019

13. __ A Banach space containing non-trivial limited sets but no non-trivial bounding set, Israel J. Math. 71, (1990), 321-327. MR 92g:46018

14. D. Leung, A Gelfand-Phillips property with respect to the weak topology, Math. Nachr. 14, (1990), 177-181. MR92j:46032

15. M. Lindström, On compact and bounding holomorphic mappings, Proc. Amer. Math. Soc. 10, (1989), 356-361. MR 89f:46103

16. - A characterization of Schwartz spaces, Math. Z. 19, (1988), 423-430. MR 89f:46009

17. M. Lindström and T. Schlumprecht, On limitedness on locally convex spaces, Arch. Math. 53, (1988), 65-74. MR 90f:46003

18. J. Mujica, Linearization of bounded holomorphic mappings on Banach spaces, Trans. Amer. Math. Soc. 32, (1991), 867-887. MR 91h:46088

19. T. Schlumprecht, A limited set that is not bounding, Proc. Roy. Irish Acad. Sect. A 90, (1990), 125-129. MR 93b:46032

Departamento de Análisis Matemático, Universidad de Valencia, Doctor Moliner 50, 46100 Burjasot (VAlencia), Spain

E-mail address: galindo@vm.ci.uv.es 